bilden: freie Medien, Meinungs- und Vereinigungsfreiheit, Streikrecht und Rechtsstaatlichkeit. Sandbrook kritisiert in diesem Kontext zu Recht die momentane Politik der Geberstaaten, die demokratische Reformen oftmals nicht mit zusätzlicher - dringend benötigter Hilfe belohnen, sondem allenf alls weniger kurzen.

Das Konzept der Zivilgesellschaft als "Motor der Demokratie" sieht der Verfasser zwar ohne praktikable Alternative, zeigt aber auch seine Grenzen auf. So ist es nicht mit dem Heranwachsen von Verbänden, Gewerkschaften etc. getan. Vielmehr müssen diese Organisationen selbst demokratische Strukturen aufweisen.

Das sozioökonomische Umfeld hält der Verfasser für ungünstig: Unteremährte Analphabeten könnten kaum eine zivile Gesellschaft aufbauen (S. 144). Hier übersieht Sandbrook, daß die Zivilgesellschaft von Eliten gebildet und geführ wird. Die Masse der armen Landbevölkerung ist von der demokratischen Transition ausgeschlossen und wird es wohl auch bleiben. Die Eliten werden höchstens versuchen, sie für ihre eigenen Ziele zu instrumentalisieren.

Die positive Korrelation, die der Verfasser zwischen Demokratisierung und Wirtschaftswachstum sieht, ist wenig uberzeugend dargestellt. Er vergleicht auf der Grundlage des Bruttosozialprodukts (einer an sich schon zweif elhaften MeBlatte) die Staaten Zaire, Niger, Ghana und Uganda mit Lesotho, Kamenm, Botswana und Mauritius für den Zeitraum 1965 - 1985 und zieht das Resümee: "Democracy and growth are thus compatible, and probably mutually reinforcing" (S. 117). Ohne die Gnondchese Sandbrooks verwerfen zu wollen, da sie in der Sache wohl zutreffend ist, ist der Vergleich problematisch. Zwar haben die genannten autoritären Regime ökonomisch sehr schlecht abgeschnitten, aber es gibt auch Ausnahmen, wenn man das Bruttosozialprodukt als MeBlatte akzeptiert: z.B. Burundi, Kenia und Mali. Die Staaten Kamerun und Lesotho gehören eher in die Kategorie der autoritären Staaten.

Insgesamt bietet das Buch einen guten Überblick über die afrikanische Wirtschaftskrise und über die Zukunftschancen durch Demokratisierung. Der Autor argumentiert praxisbezogen und illustriert seine Aussagen mit diversen Länderfallbeispielen. Auf den Afrika-Experten wirkt die Studie teilweise etwas generalisierend, aber nicht uninteressant.

Heiko Meinhardt

\title{
Martin Pabst
}

Drama Südafrika. Ein Spiel mit fünf Akteuren, vielen Statisten und einem offenen Ende

Verlagsgemeinschaft Anarche, Inning bei München, 1993, 367 S., DM 44,--

Die Verhandlungen über die Verfassung des Post-Apartheid-Südafrika sind 1993 in ein neues Stadium getreten. Die erste Zäsur stellte das Jahr 1990 dar, als Staatspräsident F.W. 
de Klerk seinen mutigen Sprung nach vome machte, die verbotenen Organisationen ANC, Kommunistische Partei (SACP) und PAC legalisierte und den über Jahrzehnte inhaftierten ANC-Führer Nelson Mandela und seine Leidensgefährten nicht nur bedingungslos freilieB, sondern sie darüber hinaus zu seinen Hauptverhandlungspartnem machte. Obwohl zwischen den wichtigsten Unterhändlern, d.s. Regienung/Nationale Partei und ANC/SACP, ein KompromiB über eine zumindest befristete Machtteilung erreicht und erste freie Wahlen zu einer Verfassunggebenden Versammlung auf den 27. April 1994 angesetzt wurden, erodiert seit gut einem Jahr die Machtbasis der bislang dominierenden Akteure.

Auf schwarzer Seite wandten sich viele enttăuschte Südafrikaner - vor allem die ca. sieben Millionen Jugendlichen aus der "lost generation" - radikalen Parolen wie dem PAC-Slogan "Ein Farmer, eine Kugel!" zu. So werden etwa bei Treffen der von Peter Mokoba geführten ANC-Jugendliga Parolen wie "Tötet den Farmer, tötet den Buren" oder "Verschwendet keine Kugeln, bewahrt sie für de Klerk auf" skandiert. Und auf weißer Seite steht eine gute Dreiviertelmehrheit heute, wie aktuelle Umfragen belegen, hinter der neuformierten Sammlungsbewegung "Afrikaner Volksfront" unter Fühning hochangesehener Generäle (Constand Viljoen, Martinus Groenewald u.a.), die einen souveränen Afrikaaner-Volksstaat anstrebt. Sie wird sich so lange an Ubergangsinstitutionen und Wahlen nicht beteiligen, ja deren Einsetzung bzw. Abhaltung notfalls durch Terror oder sogar die Auslösung eines Bürgerkrieges verhindern, solange ihr die Allianz von ANC und SACP den Volksstaat nicht zugestanden hat.

Hinzu kommt die ambivalente Rolle der von ländlichen Zulus getragenen "Inkatha Freiheits-Partei" unter Führung von Mangosuthu Buthelezi, die sich dem VerhandlungsprozeB ebenfalls verweigert und eine weitgehend autonome Provinz Natal - wenn nicht gar einen unabhängigen Staat Natal - anstrebt. Inkatha besitzt enge Kontakte zur Volksfront und droht, zusammen mit ihr den VerfassungsprozeB zu torpedieren. Beide Akteure verfügen über ein großes Machtpotential, das sich aber weniger bei Wahlen als im bewaffneten Kampf darstellen läßt.

Eine horrende Kriminalität (mindestens 50 Morde pro Tag), ein eskalierender - oft von weißer Seite geschürter - schwarz-schwarzer und neuerdings auch schwarz-weißer Bürgerkrieg und eine fatale Stagnation der Wirtschaftsentwicklung haben Südafrika 1993 an den Rand eines Desasters gebracht und seine weißen und schwarzen Bewohner tief verunsichert. Dies wurde und wird in vielen optimistisch-oberflächlichen Lageanalysen in der Regel verschwiegen. Es triumphiert ein fragwürdiger Zeitgeist!

Das Verdienst der hier zu besprechenden, gründlich recherchierten Untersuchung des Historikers Martin Pabst liegt darin, daß er alle wichtigen Beteiligten und deren Lösungsansätze vorstellt und diskutiert. Es ist ihm zuzustimmen, wenn er die Chancen einer nichtrassischen demokratischen "Neuen Nation Südafrika" mit erheblichen Fragezeichen versieht. Das fehlende Demokratieverständnis weiter Teile der schwarzen wie der weißen Bevölkerung, tribalistische Spannungen, die desolate Wirtschaftslage mit über $50 \%$ Arbeitslosigkeit sowie die nicht kompatiblen schwarzen und afrikaansen Nationalismen geben der Demokratie in Südafrika schlicht keine Chance. 
Ausführlich analysiert Pabst die Machtmittel der zentrifugalen Kräfte, die fortschreitende Etablierung peripherer Machtzentren auf Kosten der Zentralmacht, d.s. vor allem die Chaosmacht der Weißen im miliẗ̈rischen und infrastrukturellen Bereich, den wachsenden Zulauf zu paramilitärischen radikalen weißen Gruppienungen wie der faschistischen "Afrikaner Widerstandsbewegung" (AWB), das große Prestige der die Volksfront führenden Generäle im aktiven Heer, in den Kommandos der (weißen) Reservisten und der Verwaltung sowie die bedrohliche Allianz zwischen der weißen "Rechten" und unzufriedenen Zulus, die - wie die tagtäglichen Morde in den schwarzen Townships zeigen - am Tag der Wahrheit auch kämpfen würden.

Dem Konzept "Teilung des Landes", das von Pabst in seinen verschiedenen Lösungsansätzen ausführlich dargestellt wird, wird bei einer solchen Perspektive noch 1993 ein neuer Stellenwert zukommen. Landteilung wird damn nicht mehr die Träumerei ewiggestriger Afrikaaner, die in ihr eine Fortsetzung der Apartheid in neuem Gewand sehen (wie Pabst zu Recht hervorhebt, haben manche verantwortungsbewußte und realistische Afrikaanerführer wie Carel Boshoff oder Volksfrontsprecher Constand Viljoen derartige reaktionäre Ansichten in der Tat auch hinter sich gelassen!), sein, sondern - zunehmend wahrscheinlich - der letzte Ausweg, ein südafrikanisches Bosnien zu verhindern. Hierbei kommt es dann allerdings darauf an, die im ehemaligen Jugoslawien gemachten Fehler nicht zu wiederholen, sondem eine akzeptable Aufteilung Südafrikas auszuhandeln. Wichtig ist zu wissen, daß entsprechende Verhandlungen zwischen ANC/SACP, Volksfront und auch der NP bereits im August 1993 angelaufen sind. Dabei begegneten die ersteren den Fordenungen ihrer Gesprächspartner mit einem Höchstmaß an Verständnis.

Hierbei ist bemerkenswert, daß auch von liberaler Seite seit Jahrzehnten eine Landteilungsdiskussion geführt wird. Pabst macht seine Leser mit der kaum bekannten Tatsache vertraut, daß diese Diskussion - unter Teilnahme einer größeren Zahl liberaler Südafrikaner sowie einer Handvoll von Ausländern, darunter dem Rezensenten, Anfang der 70er Jahre im Haus des liberalen afrikaansen Dissidenten Van Zyl Slabbert begann. Andere prominente Liberale wie Alan Paton und Willem de Klerk (ein Bruder und enger Berater des Staatspräsidenten) haben immer wieder geäußert, daß eine "sacrificial partition", eine Teilung Südafrikas auf Kosten der Weißen, möglicherweise das letzte Mittel zur Verhinderung eines Bürgerkrieges und eine solche "Fluchtburg" einmal der letzte Ausweg für die Afrikaaner sein könne. Aus den Kreisen um Slabbert rekrutierten sich dann jene Persönlichkeiten - allen voran Abraham Viljoen -, die Vertreter der Allianz ANC/SACP und der Volksfront zu Verhandlungen zusammenführten.

Derzeit werden in Südafrika so intensiv wie nie zuvor Teilungskonzepte aufgegriffen und diskutiert, und liberale wie konservative Afrikaaner scheinen sich dabei mehr und mehr zu begegnen. Auch ein Großteil der regierenden Nationalen Partei wird sich bei der absehbaren Verschlechterung der inneren Lage sehr bald der Volksfront und ihren Zielen zuwenden. Und auf seiten des ANC gibt es hervorragende Vertreter, die bereit sind, diesen unvermeidlichen Preis für Frieden und Stabilität zu bezahlen. Sie alle werden zusammenkommen, andernfalls wird Südafrika in ein unvorstellbares Blutbad stürzen. Breyten Brey- 
tenbach sprach schon vor Jahren von der Gefahr, sein Land werde schon bald die ungezählten Varianten der Barbarei durchlaufen.

Pabsts Buch ist eine realistische Bestandsaufnahme der Situation. Im Gegensatz zu manchen anderen Südafrika-Büchem jedweder Couleur, die noch den Kategorien der Vergangenheit verhaftet scheinen, wirkt es innovativ und zukunftsorientiert. Wie er am Ende hervorhebt, ist der Ausgang des Ringens um das neue Südafrika noch völlig offen. Erst die weitere Entwicklung wird zeigen, welche der aufgezeigten Richtungen es einschlagen wird.

Erdnann Klaus Frhr. von der Ropp

\section{Nikolaus Werz}

Das neuere politische und soxialwissenschaftiche Denken in Lateinamerika

Amold-Bergstraesser-Institut, Freiburg, 1991, 424 S., DM 29,--

Seine Habilitationsschrift zum neueren Denken in Lateinamerika leitet der Freiburger Politikwissenschaftler Nikolaus Werz in eigentümlicher Weise ein: Er betont besonders, was die vorliegende Studie nicht leisten soll oder kamn. So soll eine ideologiekritische Analyse der untersuchten Texte nicht stattfinden, statt dessen der Selbstreflexion lateinamerikanischer Intellektueller und Politiker Raum gegeben werden. Politisch-ideologische Strömungen, wie sie sich u.a. in politischen Parteien kristallisiert haben, werden nicht betrachtet. Statt dessen soll sich die Untersuchung vor allem an bestimmten Begriffen der zeitgenössischen Debatte (Nationalismus, Modernisienung, Abhängigkeit, Befreiung) orientieren. Auch eine Ideengeschichte Lateinamerikas, so Werz, habe er nicht geschrieben, vielmehr bestimmte geschichtliche Perioden und thematische Zusammenhänge ausgewählt. Ziel der Arbeit sei die "Aufarbeitung wichtiger Merkmale des politischen und kulturellen Selbstverständnisses in Lateinamerika" (S. 8).

Angesichts der Breite des gesetzten Themas bieten sich zwei Herangehensweisen an: Erstens kömnte der Autor einen thematisch begrenzten Zugriff wählen und die Arbeit z.B. anhand bestimmter Leitbegriffe (wie sie Werz ja auch erwähnt) strukturieren. Eine Alternative bestünde zweitens darin, die als wesentlich identifizierten Denkansätze in historischer Perspektive darzustellen und jeweils für sich genommen zu diskutieren. Werz hat sich für diese zweite Vorgehensweise entschieden, gleichzeitig aber auch eine thematische Gliederung vorgenommen. Hierin drückt sich anscheinend der Wunsch aus, eine subjektive, thematisch eingeschränkte Beurteilung des lateinamerikanischen Denkens zu vermeiden und die behandelten Strömungen und Autoren 'zu ihrem Recht kommen' zu lassen. Dementsprechend stehen hermeneutische Methoden im Mittelpunkt der Arbeit. Allerdings hat sich Werz damit auf die Herkules-Arbeit einer übergreifenden und angesichts der Fülle des Stoffes kaum zu leistenden Bestandsaufnahme eingelassen. 